\title{
Neuroprotective effects of Ilexonin A following transient focal cerebral ischemia in rats
}

\author{
AI-LING XU ${ }^{1,2}$, GUAN-YI ZHENG ${ }^{1}$, ZHI-JIAN WANG ${ }^{1,3}$, XIAO-DONG CHEN $^{4}$ and QIONG JIANG ${ }^{4}$ \\ ${ }^{1}$ Department of Traditional Chinese Medicine, The Affiliated Union Hospital of Fujian Medical University, Fuzhou, Fujian 350001; \\ ${ }^{2}$ Neonatal Department, The People's Hospital Affiliated to Fujian University of Traditional Chinese Medicine, \\ Fuzhou, Fujian 350004; ${ }^{3}$ Department of Neurology, Fuzhou Neuro-Psychiatric Hospital, Fuzhou, Fujian 350000; \\ ${ }^{4}$ Burns Institute of the Affliated Union Hospital of Fujian Medical University, Fuzhou, Fujian 350001, P.R. China
}

Received March 24, 2015; Accepted December 30, 2015

DOI: $10.3892 / \mathrm{mmr} .2016 .4921$

\begin{abstract}
Ilexonin A is a compound isolated from the root of a plant commonly used in traditional Chinese medicine. The aim of the present study was to investigate the possible protective mechanism of Ilexonin A in rats subjected to occlusion of the middle cerebral artery (MCAO). Transient focal cerebral ischemia was induced by $2 \mathrm{~h}$ of MCAO, followed by reperfusion. Ilexonin A at doses of 20,40 and $80 \mathrm{mg} / \mathrm{kg}$ were administered via intraperitoneal injection immediately following ischemia/reperfusion. The expression levels of glial fibrillary acidic protein (GFAP), ionized calcium-binding adapter molecule-1 (Iba-1), vascular endothelial growth factor (VEGF), fetal liver kinase-1 (Flk-1) and Nestin were examined using immunostaining and Western blot analysis of the peri-infarct region following ischemia/reperfusion. Ilexonin A significantly decreased the infarct volume and improved neurological deficits in a dose-dependent manner. The expression levels of VEGF, Flk-1 and Nestin were significantly increased in the rats treated with Ilexonin A, compared with the rats administered with saline. Following treatment with Ilexonin A, a higher number of GFAP-positive astrocytes were found in the Ilexonin A-treated rats at 1,3 and 7 days, compared with the rats exposed to ischemia only, however, there were fewer astrocytes at 14 days, compared with the ischemia group. Ilexonin A significantly decreased the protein expression of Iba-1. The results of the present study suggested that the protective effects of Ilexonin A were associated with revascularization, neuronal regeneration, and the regulation of astrocyte and microglia cell activation.
\end{abstract}

Correspondence to: Dr Guan-Yi Zheng, Department of Traditional Chinese Medicine, The Affiliated Union Hospital of Fujian Medical University, 29 Xinquan Road, Gulou, Fuzhou, Fujian 350001, P.R. China

E-mail: guanyi.zheng@yahoo.com

Key words: Ilexonin A, revascularization, transient focal cerebral ischemia, neuroprotection

\section{Introduction}

Although stroke is one of the leading contributors to mortality and long-term disability rates among adults around the world, innovative neuroprotective treatments for therapy following a stroke require further investigation. An area of investigation, which is gaining increasing attention is the use of herbal medicines or their extracts for treating stroke. Ilexonin A, a 3 $\beta$-succinyl-18-dehydro-disodic ursolate (Fig. 1), is a compound extracted from the herb, Ilex pubescens, which shows good therapeutic effects when used for the treatment of coronary artery disease, angina and vasculitis $(1,3)$. It has been confirmed that the therapeutic effects of Ilexonin A are achieved through improving blood circulation via its antithrombotic and anti-inflammatory activities (4). As early as 1985, Luo et al found that dysfunction induced by cerebral ischemia was ameliorated following treatment with Ilexonin A. Evidence obtained in subsequent studies has suggested that Ilexonin $\mathrm{A}$, which can reduce infarct volume and improve neurological deficit, has a neuroprotective potential following cerebral ischemia/reperfusion injury, the effect of which is partly attributed to enhancement of the secretion of neurotrophic factors, mitigating cerebral edema and promoting neural regeneration $(5,6)$.

Astrocytes and microglia cells reside in the central nervous system (CNS) as ubiquitously distributed quiescent cell populations, which are able to in change morphology, number and function when activated by ischemic conditions $(7,8)$. It has been suggested that reactive astrocytes and microglia are multifunctional cells, which, depending on the microenvironment, can be either beneficial or detrimental following ischemia/reperfusion $(9,10)$. Previous reports have suggested that reactive astrocytes and microglia can promote tissue integrity, seal off injured tissue and restrict neuronal death through cell proliferation (11), phagocytosis of cellular debris and the secretion of neurotrophic factors $(11,12)$. However, they can also mediate brain edema and inflammation by producing neurotoxic substances and pro-inflammatory cytokines following ischemic injury $(13,14)$. The present study aimed to investigate the characteristics of activated astrocytes and microglia following the post-ischemia administration of Ilexonin A. 
It has been suggested that angiogenesis and neurogenesis occurring in the peri-infarct region are associated with long-term recovery $(15,16)$; and the two processes appear to be governed by ischemia-induced growth factors. One of the most important neurotrophic and angiogenic factors found during stroke recovery, which is fundamental for adult angiogenesis, is vascular endothelial growth factor (VEGF) (17). A previous study suggested that VEGF mediates neuroprotection and promotes neural regeneration when it is bound by its specific receptor, fetal liver kinase 1 (Flk-1) (18). Endogenous neurogenesis and neovascularization are substantially activated and occur in close proximity to the ipsilateral neocortex of the ischemic brain (15).

The present study was performed in order to explore the underlying protective mechanisms of Ilexonin A in rats, and to provide a reliable scientific basis for treating ischemic cerebrovascular disease with Ilexonin A. The current study investigated the effects of Ilexonin A on the expression levels of VEGF, Flk-1 and Nestin in the peri-infarct region following transient focal cerebral ischemia.

\section{Materials and methods}

Reagents. All reagents were purchased from Beijing Zhongshan Biotechnology Co., Ltd. (Beijing, China), unless otherwise stated. Ilexonin A was supplied by Guangdong Boro Pioneer Pharmaceutical Group Co., Ltd. (Guangdong, China; approval no. Z44023366).

Animal treatment and administration. A total of 108 male Sprague-Dawley rats (Grade II) weighing $250 \pm 10 \mathrm{~g}$, aged 6-8 weeks, were supplied by Shanghai SLAC Laboratory Animal Co. Ltd (Shanghai, China; certificate no. 2007-0005). The rats were housed in temperature $\left(22-25^{\circ} \mathrm{C}\right)$ and humidity (40-70\%) controlled conditions with a $12 \mathrm{~h}$ light/dark cycle and ad libitum access to food and water. The study was approved by the ethics committee of The Affiliated Union Hospital of Fujian Medican University (Fuzhou, China). The rats were divided into six groups for investigation: Normal group; Sham group, Ischemia group, and Ilexonin A groups receiving 20, 40 , and $80 \mathrm{mg} / \mathrm{kg}$, respectively. Each group was divided into four subgroups $(n=6)$, based on the onset of reperfusion, set at $1,3,7$ or 14 days following ischemia. The normal control rats were injected with equal volumes of saline.

Focal cerebral ischemia/reperfusion procedure. Focal cerebral ischemia/reperfusion injury was induced by middle cerebral artery occlusion (MCAO), based on the method developed by Longa et al (19). The rats were anesthetized with an intraperitoneal injection of $10 \%$ chloral hydrate $(300 \mathrm{mg} / \mathrm{kg}$; Tianjin Kemiou Chemical Reagent Co., Ltd., Tianjin, China). The left common carotid artery, external carotid artery (ECA) and internal carotid artery (ICA) were isolated via blunt dissection following a ventral midline incision of the neck. The branches of the ECA were cut off, and the whole of the ECA was ligated and dissociated at the distal end by electric coagulation. A monofilament nylon suture $(0.26 \mathrm{~mm}$; Beijing Zhongshan Biotechnology Co., Ltd.), with its tip rounded by paraffin (Beijing Donglin Changsheng Biotechnology Co., Ltd., Beijing, China), was inserted $18 \pm 0.5 \mathrm{~mm}$ into the ICA

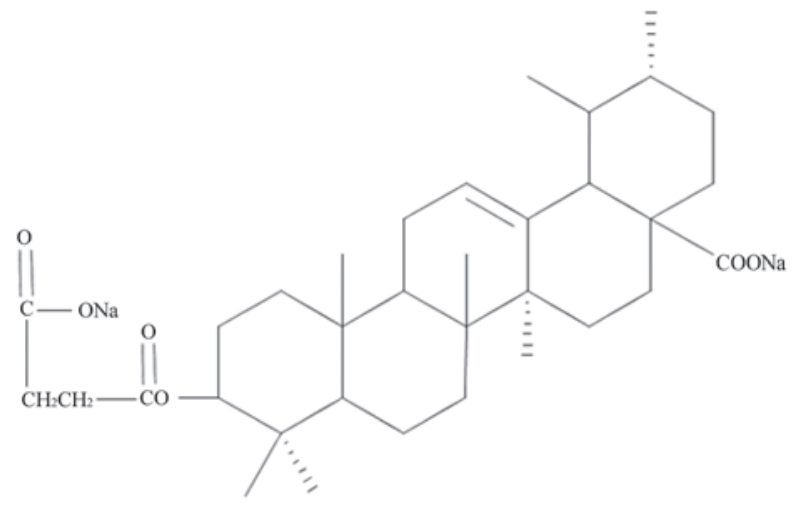

Figure 1. Structure of Ilexonin A.

from the ECA, to occlude the origin of the MCA. The suture was fixed and the incision was closed. Following $2 \mathrm{~h}$ of ischemia, the nylon suture was withdrawn to allow reperfusion. The rats in the normal group did not undergo surgery. The sham-operated rats underwent identical surgery, with the exception that the nylon suture was not inserted.

Inclusion criteria of MCAO and neurological severity scoring of animals. Following recovery of the rats from anesthesia, neurological severity was evaluated for the determination of MCAO using a five point score, according to Longa's method (19): 0 , no deficit; 1 , failure to extend right forelimb fully; 2 , circling to the right; 3 , falling down to the right; 4 no spontaneous walking with a depressed level of consciousness. Achievement of MCAO was confirmed using a score of 1-3 points. The rats were re-evaluated using the same method prior to sacrifice by anaesthetic overdose using $10 \%$ chloral hydrate.

Tetrazolium chloride staining. The rats were anesthetized and received cardiac perfusion with $100 \mathrm{ml}$ saline, followed by $200 \mathrm{ml}$ 4\% paraformaldehyde (Beijing Donglin Changsheng Biotechnology Co.., Ltd.) in 0.1 M phosphate buffer (pH 7.4). The brains were carefully removed when the liver and extremities turned white in color. The brains were frozen at $-20^{\circ} \mathrm{C}$ for $20 \mathrm{~min}$, and then cut from the anterior pole into five coronal slices of $2 \mathrm{~mm}$ thickness. The slices were stained with $2 \% 2$, 3, 5-triphenyltetrazolium chloride solution (Sigma-Aldrich, St. Louis, MO, USA) in the dark at $37^{\circ} \mathrm{C}$ in an incubator for $30 \mathrm{~min}$, and turned over every $5 \mathrm{~min}$. A $10 \%$ buffered-formalin solution (Beijing Donglin Changsheng Biotechnology Co.., Ltd.) was used for fixation $(24 \mathrm{~h})$ prior to imaging with a digital camera (VPC-SH1; Sanyo Electric Co., Ltd., Osaka, Japan). The normal brain tissue was stained red, whereas the ischemic area remained unstained.

Immunohistochemical examination. The rats were anesthetized and perfused in the same manner as that described above. The brains were removed and post-fixed for $24 \mathrm{~h}$ in the same fixative solution, and dehydrated in a sucrose gradient $(15,20$ and 30\%) in $0.1 \mathrm{M}$ phosphate buffer until they sank to the bottom. Subsequently, $8 \mu \mathrm{m}$ sections were cut using a cryomicrotome (Microm HM525; Thermo Fisher Scientific, Inc., Waltham, MA, USA) for use in immunohistochemical probing. 
Table I. Neurological severity scores in each group.

\begin{tabular}{lcccr}
\hline Group & 1 day post-MCAO & 3 days post- MCAO & 7 days post-MCAO & 14 days post-MCAO \\
\hline Normal & 0 & 0 & 0 & 0 \\
Sham & 0 & $0.17 \pm 0.41$ & 0 & 0 \\
Ischemia & $2.67 \pm 0.52$ & $2.33 \pm 0.52$ & $2.00 \pm 0.63$ & $1.50 \pm 0.55^{\mathrm{a}}$ \\
IA20 $\mathrm{mg} / \mathrm{kg}$ & $2.33 \pm 0.52$ & $1.67 \pm 0.52^{\mathrm{a}}$ & $1.33 \pm 0.52^{\mathrm{a}}$ & $1.17 \pm 0.75$ \\
IA40 $\mathrm{mg} / \mathrm{kg}$ & $2.00 \pm 0.63$ & $1.50 \pm 0.55$ & $1.33 \pm 0.52^{\mathrm{a}}$ & $0.67 \pm 0.52^{\mathrm{a}}$ \\
IA80 $\mathrm{mg} / \mathrm{kg}$ & $2.16 \pm 0.41$ & $1.67 \pm 0.52^{\mathrm{a}}$ & $0.83 \pm 0.41^{\mathrm{a}}$ \\
\hline
\end{tabular}

Values are presented as the mean \pm standard deviation $(n=6)$. Statistical differences were determined using one-way analysis of variance. ${ }^{\mathrm{a}} \mathrm{P}<0.05$, compared with the ischemia group. MCAO, middle cerebral artery occlusion; IA, Ilexonin A.

Immunohistochemistry (IHC) was performed in strict accordance with the datasheet of the Elivision kit (Fuzhou Maixin Biotechnology Development Co., Ltd., Fuzhou, China), with rabbit polyclonal anti-glial fibrillary acidic protein (GFAP) antibody (1:5,000; ab7260; Abcam, Cambridge, UK) to identify astrocytes, goat polyclonal anti-ionized calcium-binding adapter molecule-1 (Iba-1) antibody (1:100; ab5076; Abcam) to identify microglia, and mouse monoclonal anti-Nestin antibody (1:100; sc-33677; Santa Cruz Biotechnology, Inc., Dallas, TX, USA) to identify neural progenitor cells. In the negative controls, 0.01 M PBS was substituted for the primary antibody. The results were visualized using a 3, 3'-diaminobenzidine kit (Beijing Zhongshan Golden Bridge Biotechnology Co., Ltd, Beijing, China). A microscope (CX40; Olympus Corporation, Tokyo, Japan) was used for image acquisition. The positive cells in each section were evaluated using Image Pro Plus 5.0 (Media Cybernetics, Inc., Rockville, MD, USA) in five high power microscopic fields (CX40; Olympus Corporation, Tokyo, Japan; magnification, $\mathrm{x} 400)$.

Western blot analysis. The rats were infused with 100-200 ml saline following anesthesia and the brains were removed. The peri-infarct region was disassociated and lysed in $10 \mu \mathrm{l} / \mu \mathrm{g}$ radioimmunoprecipitation assay lysis buffer with $0.01 \mathrm{M}$ phenylmethanesulfonylfluoride (both from Beyotime Institute of Biotechnology, Haimen, China). The samples were homogenized by sonication and centrifuged at $14,000 \mathrm{x} \mathrm{g}$ at $4^{\circ} \mathrm{C}$ for five min, following which the supernatant was collected. A bicinchoninic acid protein assay kit (P0010S; Beyotime Institute of Biotechnology) was used to quantify protein concentrations. The protein samples were boiled for $5 \mathrm{~min}$, and $20 \mu \mathrm{g}$ was loaded onto SDS-PAGE gels (12\% for Iba-1, GFAP and VEGF; 6\% for Nestin and Flk-1). Following electrophoresis, the proteins were electrically transferred onto a nitrocellulose membrane (pore size, $0.45 \mu \mathrm{m}$; EMD Millipore, Billerica, MA, USA). The membranes were blocked with $5 \%$ nonfat milk in Tris-buffered saline with tween (TBST) containing Tris- $\mathrm{Hcl}(0.1 \mathrm{M} ; \mathrm{pH} 7.5) 0.9 \,% \mathrm{NaCl}$ and $0.05 \%$ Tween-20, at room temperature for $1 \mathrm{~h}$ on a shaking table. The membranes were subsequently incubated with the following primary antibodies in TBST at $4{ }^{\circ} \mathrm{C}$ overnight: Rabbit polyclonal anti-GFAP, (1:20,000; ab7260), goat polyclonal anti-Iba-1 (1:200; ab5076; both purchased from Abcam), mouse monoclonal anti-Nestin (1:300), mouse monoclonal
anti-VEGF (1:200; sc-7269), mouse monoclonal anti-Flk-1 $(1: 200 ;$ sc-6251) and mouse monoclonal anti- $\beta$-actin $(1: 2,000$; sc-47778) (all obtained from Santa Cruz Biotechnology, Inc.).Following being washed with TBST, the membranes were incubated with secondary antibodies (1:6,000; Beijing Zhongshan Golden Bridge Biotechnology, Co., Ltd.) at room temperature for $2 \mathrm{~h}$ and washed with TBST. The immunoblots were visualized using enhanced chemiluminescence detection reagents (KPL, Gaithersburg, MD, USA). Densitometry was performed using ImageMaster ${ }^{\circledR}$ VDS gel imaging and analysis system (AlphaImager 2200; Alpha Innotech Corporation Company, San Jose, CA, USA), with $\beta$-actin as the loading control.

Statistical analysis. All data are expressed as the mean \pm standard deviation and were analyzed using one-way analysis of variance using the SPSS 17.0 Software package (SPSS, Inc., Chicago, IL, USA). The differences between the groups were analyzed using the least significant difference test for homogeneity of variance and the Games-Howell test for heterogeneity of variance. $\mathrm{P}<0.05$ was considered to indicate a statistically significant difference.

\section{Results}

Tetrazolium chloride staining. The induction of ischemic infarction by MCAO involves the striatum and cortex in shorter time spans and other regions at later time points (20). In the present study, brain atrophy induced by tissue degeneration and necrosis was observed in the ischemic core. Following treatment with Ilexonin A, the infarct volume was decreased, particularly at 7 days, in the Ilexonin A $40 \mathrm{mg} / \mathrm{kg}$ group (Fig. 2).

Effect of Ilexonin A on neurological deficits. Neurological symptoms, including unconsciousness, ptosis and tonic seizures, were exhibited on day 1 in the rats subjected to MCAO, with or without administration of Ilexonin A. With prolongation of reperfusion, the neurological deficits gradually disappeared, and rats treated with Ilexonin A recovered at a faster rate, compared with those in the ischemia group (Table I).

Effects of Ilexonin A on astrocyte activation. In the IHC rat brain sections, GFAP-positive cells were observed sporadically, primarily in the cortex, in the normal and sham groups, whereas 
A

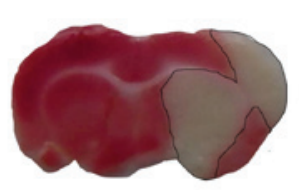

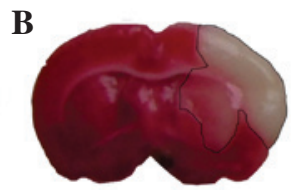

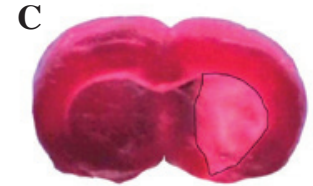

Figure 2. IA decreases infarct volume following ischemia/reperfusion. Normal brain tissue was stained red, whereas ischemic area remained unstained, (A) MCAO 7 days; (B) MCAO 7 days+IA 40 mg/kg; (C) MCAO 14 days+IA 40 mg/kg. MCAO, middle cerebral artery occlusion; IA, Ilexonin A.

A

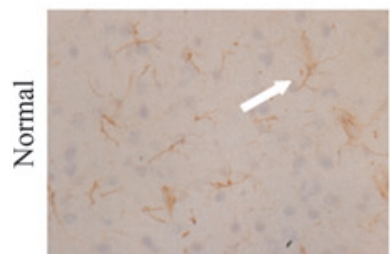

a

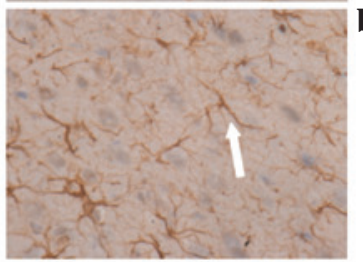

c

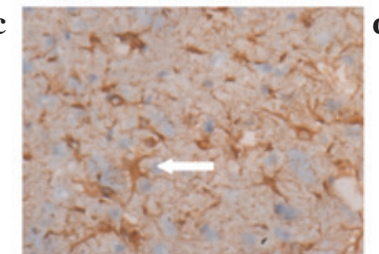

e

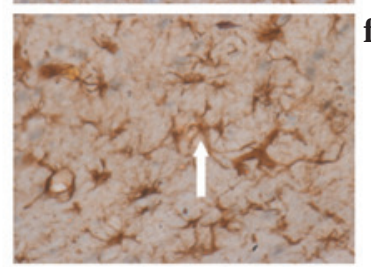

g

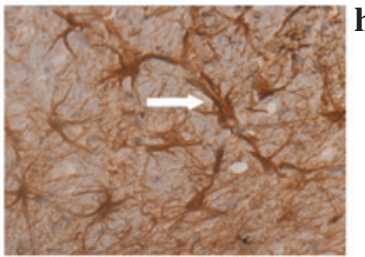

Ischemia
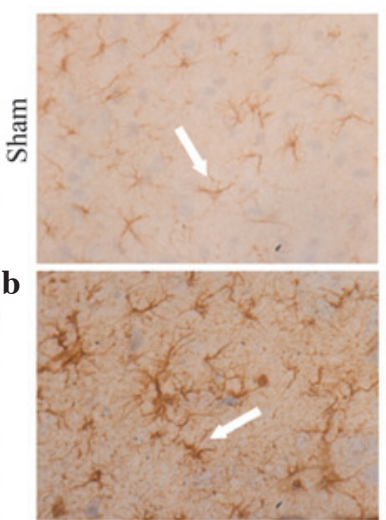

d
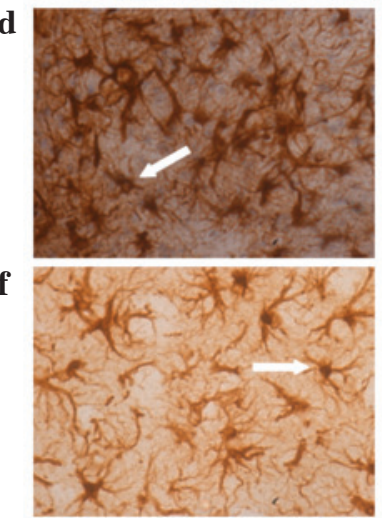

h

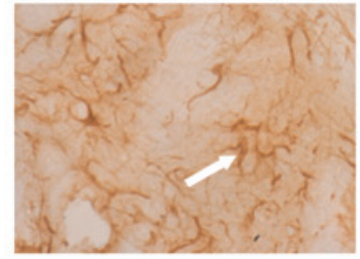

IA $40 \mathrm{mg} / \mathrm{kg}$

Peri-infarct

B

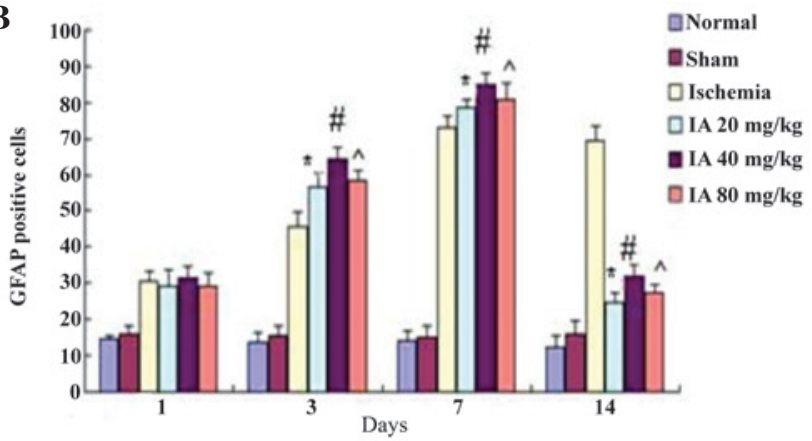

C

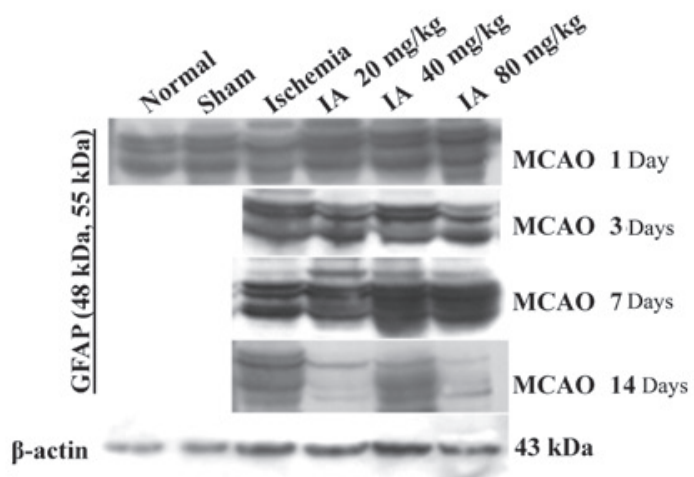

D

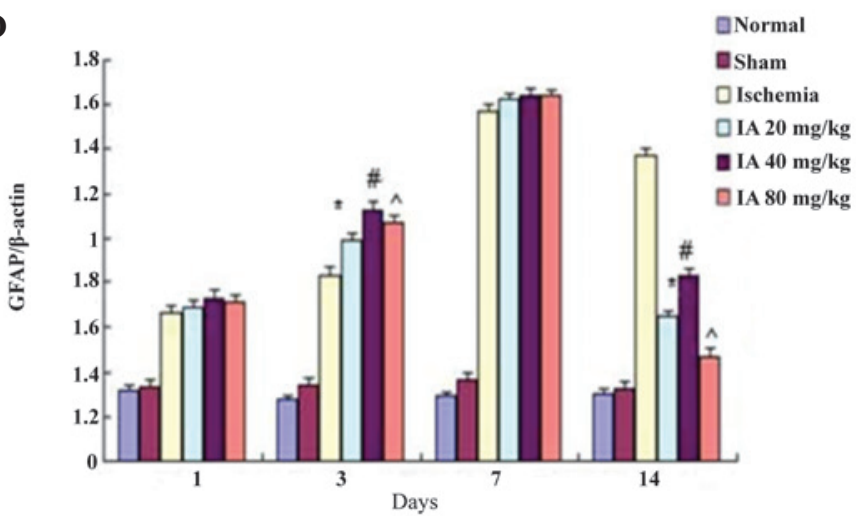

Figure 3. Effect of Ilexonin A on the activation of astrocytes. Immunostaining for GFAP in the peri-infarct region at different time points following ischemia/reperfusion. (A) Ischemia group (a) 1, (c) 3, (e) 7 and (g) 14 days after MCAO; and the administration of $40 \mathrm{mg} / \mathrm{kg}$ IA (b) 1, (d) 3, (f) 7 and (h) 14 days after MCAO. Astrocytes were stained brown (arrow). Magnification, x400; scale bar=50 $\mu \mathrm{m}$. (B) GFAP-positive cells at different doses of IA. Protein expression levels of GFAP were detected using (C) Western blot analysis, and (D) quantification of the results was performed using $\beta$-actin protein as a loading control. Values are expressed as the mean \pm standard deviation $(\mathrm{n}=6) .{ }^{*} \mathrm{P}<0.05,{ }^{\#} \mathrm{P}<0.05$ and ${ }^{\wedge} \mathrm{P}<0.05$, vs. ischemia group. MCAO, middle cerebral artery occlusion; IA, Ilexonin A; GFAP, glial fibrillary acidic protein.

their numbers were markedly increased in the peri-infarct region following ischemia/reperfusion (Fig. 3A). Over time, the GFAP-positive cells in the ischemia group became markedly activated, with a marked change in morphology, which included swelling of the soma, darkening of the cytoplasm and growth and thickening of cell processes, which even interlaced with each other, like a network, at day 14. Compared with the ischemia group, an increase in GFAP-immunoreactivity was observed from day 1 following ischemia/reperfusion in the Ilexonin A groups, persisting until day 7 (Fig. 3B). Of note, the GFAP-immunoreactivity decreased per contra 14 days following ischemia/reperfusion in all Ilexonin A groups, and the morphology was altered less, particularly in the $40 \mathrm{mg} / \mathrm{kg}$ dose Ilexonin A group. 
A

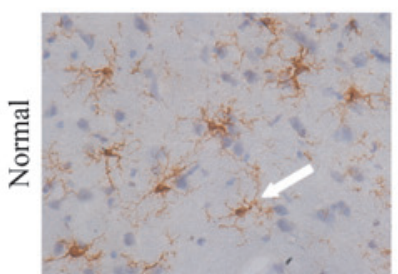

a

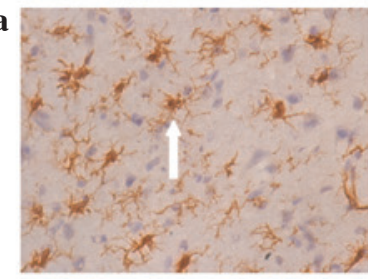

c

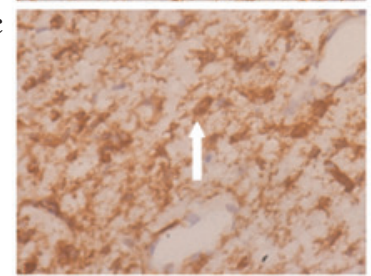

e

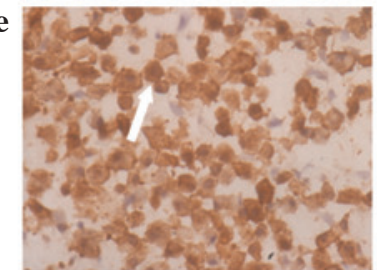

g

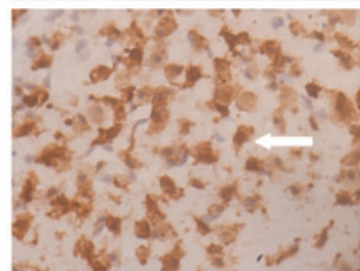

Ischemia

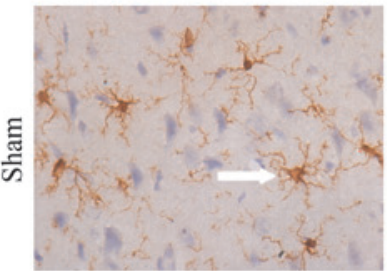

b

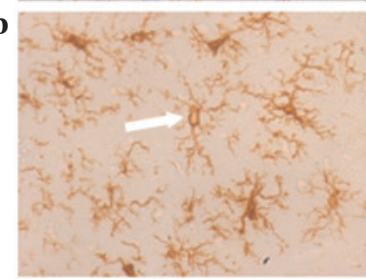

d

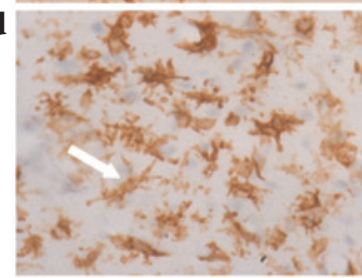

f

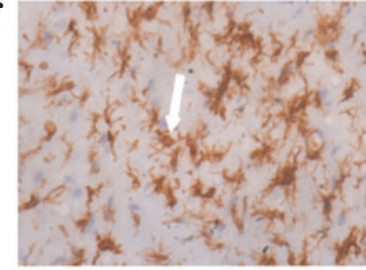

h

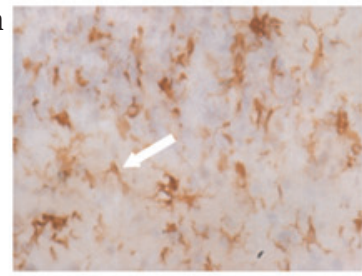

IA $40 \mathrm{mg} / \mathrm{kg}$

Peri-infarct

B

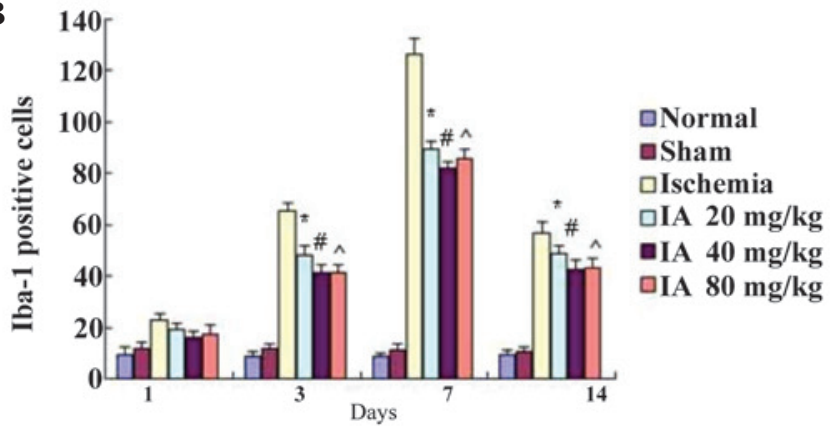

C
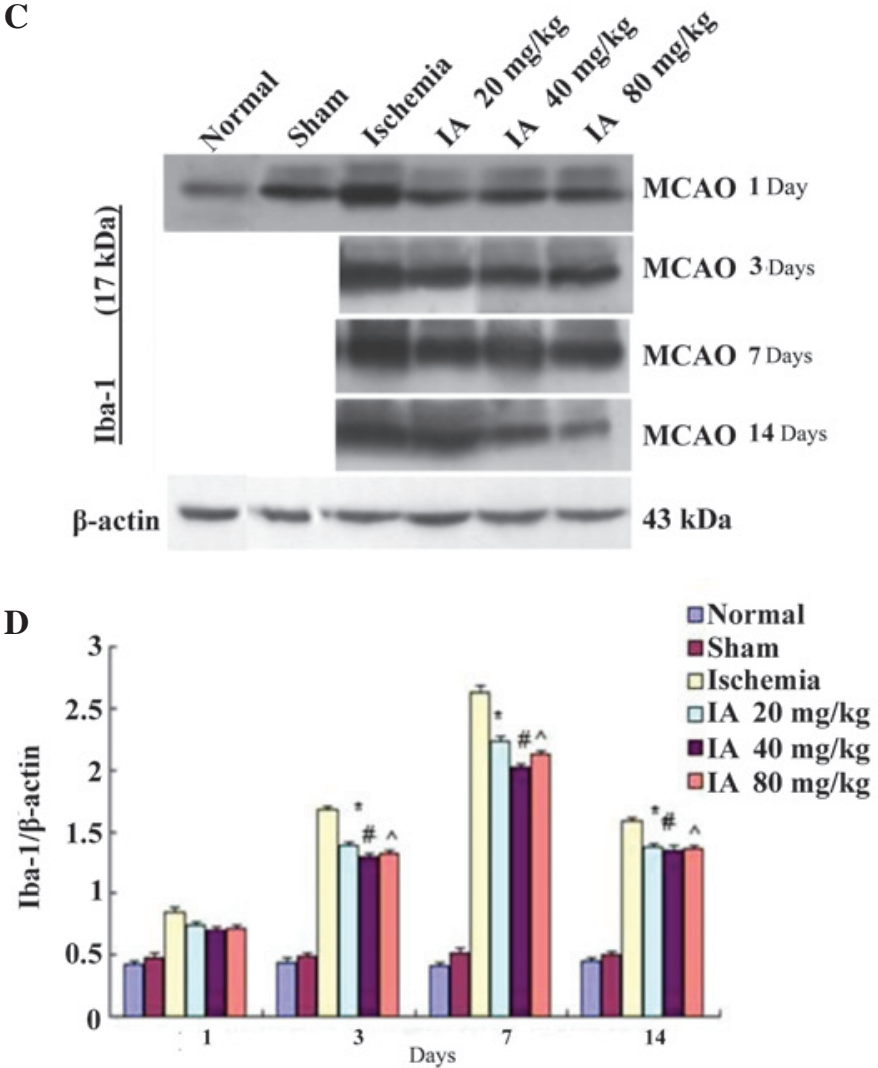

Figure 4. Effect of IA on the activation of microglia. Immunostaining for Iba-1 in the peri-infarct region at different time points following ischemia/reperfusion. (A) Ischemia group (a) 1, (c) 3, (e) 7 and (g) 14 days after MCAO; and the administration of $40 \mathrm{mg} / \mathrm{kg} \mathrm{IA} \mathrm{(b)} \mathrm{1,} \mathrm{(d)} \mathrm{3,} \mathrm{(f)} 7$ and (h) 14 days after MCAO (B) Iba-1-positive cells at different doses of IA. Microglia are stained brown (arrow). Magnification, $\mathrm{x} 400$; scale bar=50 $\mu$ m. Protein expression levels of Iba-1 were detected using (C) Western blot analysis and (D) quantification of results was performed using $\beta$-actin as a loading control. Values are expressed as the mean \pm standard deviation $(\mathrm{n}=6) .{ }^{*} \mathrm{P}<0.05,{ }^{\#} \mathrm{P}<0.05$ and ${ }^{\wedge} \mathrm{P}<0.05$, vs. ischemia group. $\mathrm{MCAO}$, middle cerebral artery occlusion; IA, Ilexonin $\mathrm{A}$; Iba-1, ionized calcium-binding adapter molecule-1.

Following reperfusion, the expression level of GFAP in the peri-infarct region was markedly increased, and reached a peak at day 7 in the ischemia and Ilexonin A group, as demonstrated by Western blot analysis (Fig. 3C). Compared with the ischemia group, the levels of GFAP in the Ilexonin A group were higher on days 1, 3 and 7, however, there were fewer GFAP-positive cells on day 14, compared with the ischemia rats (Fig. 3D).

Effect of Ilexonin A on the activation of microglia. The IHC results revealed no significant difference in the numbers of Iba-1-positive cells between the normal and sham groups, with only quiescent microglia in the peri-infarct region. In the ischemia group, the number of Iba-1-positive cells was increased between days 1 and 14, peaking 7 days post-ischemia/reperfusion (Fig. 4A and B). The quiescent microglia, which had elliptic and small soma, and more cell processes, gradually changed into rod-like cells, which had enlarged soma and thicker, shorter processes. These finally changed to amoeba-like macrophages, with large rounded soma and fewer or no cell processes. The numbers of Iba-1 positive cells, particularly the round macrophage-like cells, in the Ilexonin A groups were significantly reduced at each time point, compared with the ischemia group. The change was most marked at 7 days post-ischemia/reperfusion in the $40 \mathrm{mg} / \mathrm{kg}$ Ilexonin A group.

As shown in Fig. 4, compared with the normal and sham groups at time points $>1$ day, cerebral ischemia in the rats subjected to MCAO and Ilexonin A treatment significantly induced the expression of Iba-1, as detected using Western blot analysis (Fig. 4C and D). The expression of Iba-1 peaked 7 days following reperfusion in the ischemia and Ilexonin A 
A

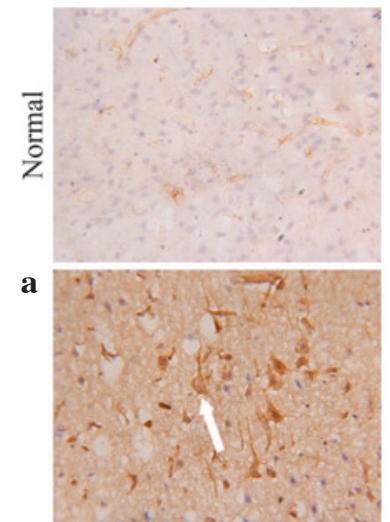

c

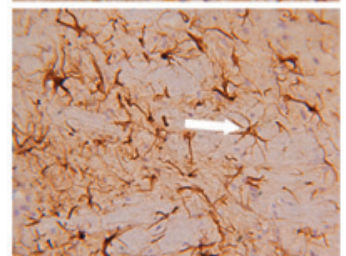

e

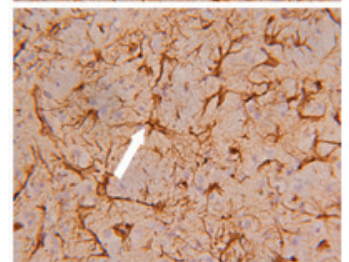

g

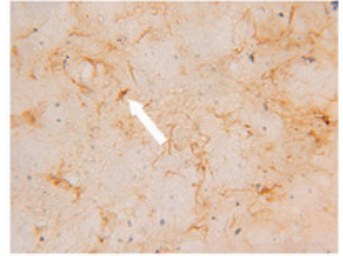

Ischemia
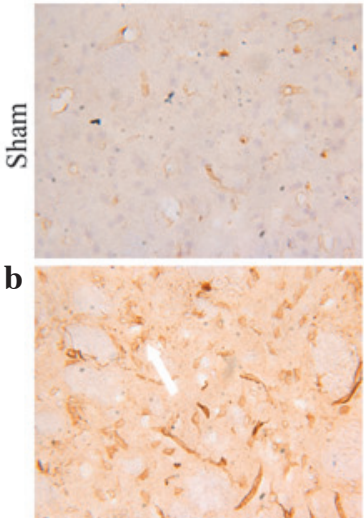

d

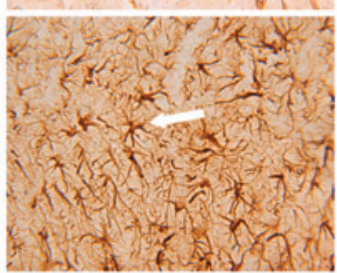

f

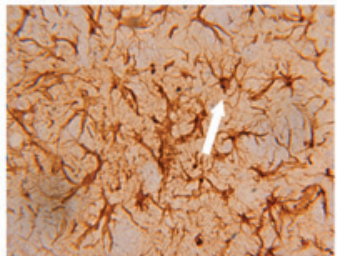

h

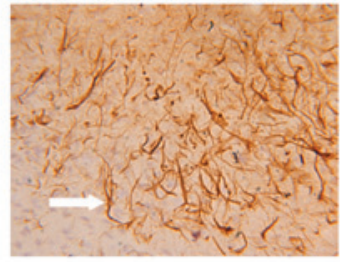

IA $40 \mathrm{mg} / \mathrm{kg}$

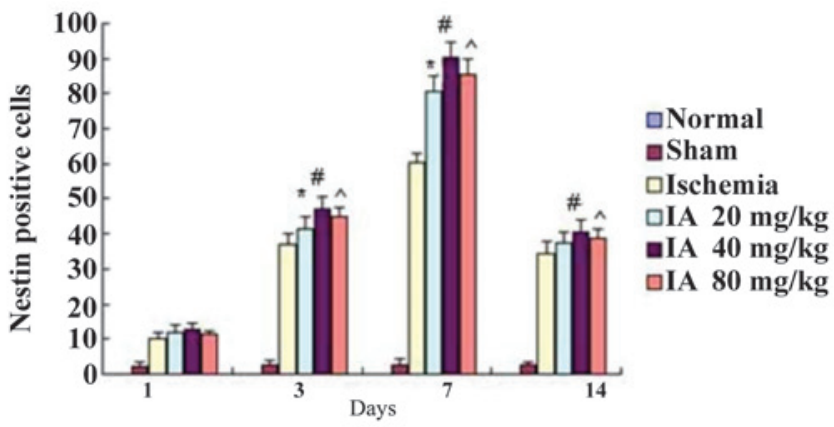

C

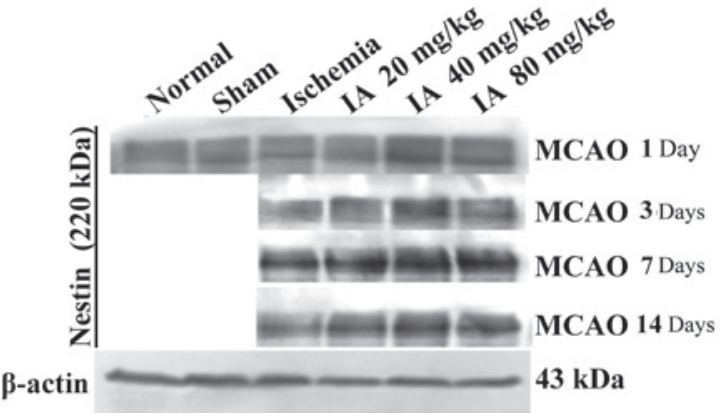

D

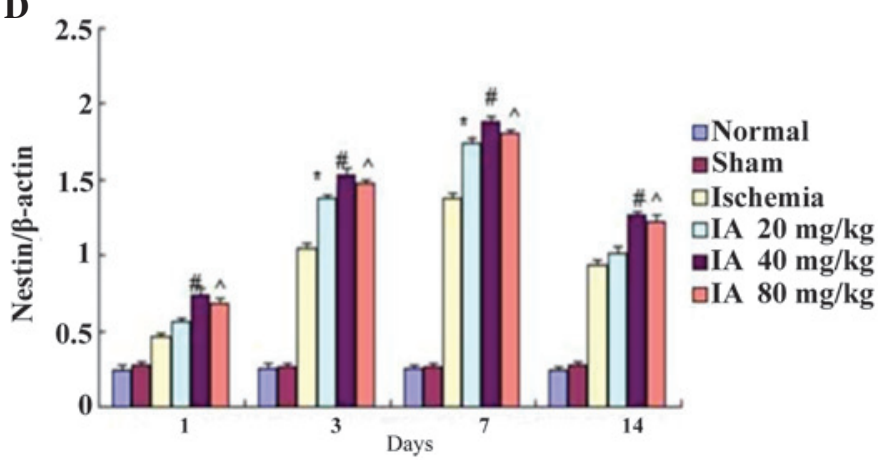

Figure 5. IA increases proliferation of neural stem cells. Immunostaining for Nestin in the peri-infarct region at different time points following ischemia/reperfusion. (A) Ischemia group (a) 1, (c) 3, (e) 7 and (g)14 days after MCAO; and the administration of 40 mg/kg IA (b) 1, (d) 3, (f) 7 and (h) 14 days after MCAO. (B) Nestin-positive cells at different doses of IA. Neural stem cells were stained brown (arrow). Magnification, $\mathrm{x} 400$; scale bar=50 $\mu \mathrm{m}$. Protein expression levels of Nestin were detected using (C) Western blot analysis and (D) quantification of results was performed using $\beta$-actin as a loading control. Values are expressed as the mean \pm standard deviation $(\mathrm{n}=6)$. ${ }^{*} \mathrm{P}<0.05,{ }^{\#} \mathrm{P}<0.05$ and ${ }^{\wedge} \mathrm{P}<0.05$, vs. ischemia group. MCAO, middle cerebral artery occlusion; IA, Ilexonin A.

groups. Compared with the ischemia group, Ilexonin A treatment significantly decreased the expression of Iba-1, compared with the group exposed to reperfusion alone, particularly in the $40 \mathrm{mg} / \mathrm{kg}$ Ilexonin A group.

Effect of Ilexonin A on the proliferation of neural stem cells. In the normal and sham groups, a number of small vessels and micro-vascular endothelial cells were weakly positive for Nestin. Following ischemia/reperfusion, the numbers of Nestin-positive cells around the ischemia core increased significantly on days 3 , 7 and 14, with a peak at 7 days, in the ischemia and Ilexonin A groups (Fig. 5A and B). In the rats subjected to MCAO treatment, a significant induction of Nestin was observed in the peri-infarct region. A further increase was observed in all Ilexonin A groups (Fig. 5C and D).

Effect of Ilexonin A on the expression of VEGF. Compared with the normal and sham group, the expression of VEGF was increased at day 1 post-ischemia/reperfusion, and was highest at 7 days. The expression of VEGF was further increased in the rats receiving Ilexonin A at each time point, particularly at 7 days in the $80 \mathrm{mg} / \mathrm{kg}$ group (Fig. 6).

Effect of Ilexonin A on the expression of Flk-1. The expression levels of Flk-1 were increased 3 days following ischemia/reperfusion, and reached a peak at 7 days in the ischemia group, compared with the normal and sham groups. Following treatment with Ilexonin A, the expression levels of Flk-1 were further increased at each time point, particularly at 7 days in the $80 \mathrm{mg} / \mathrm{kg}$ Ilexonin A group (Fig. 7).

\section{Discussion}

Ilexonin A is a compound, which is extracted from the herb, Ilex pubescens, and has been shown to have antithrombotic effects, anti-inflammatory effects and to improve blood 

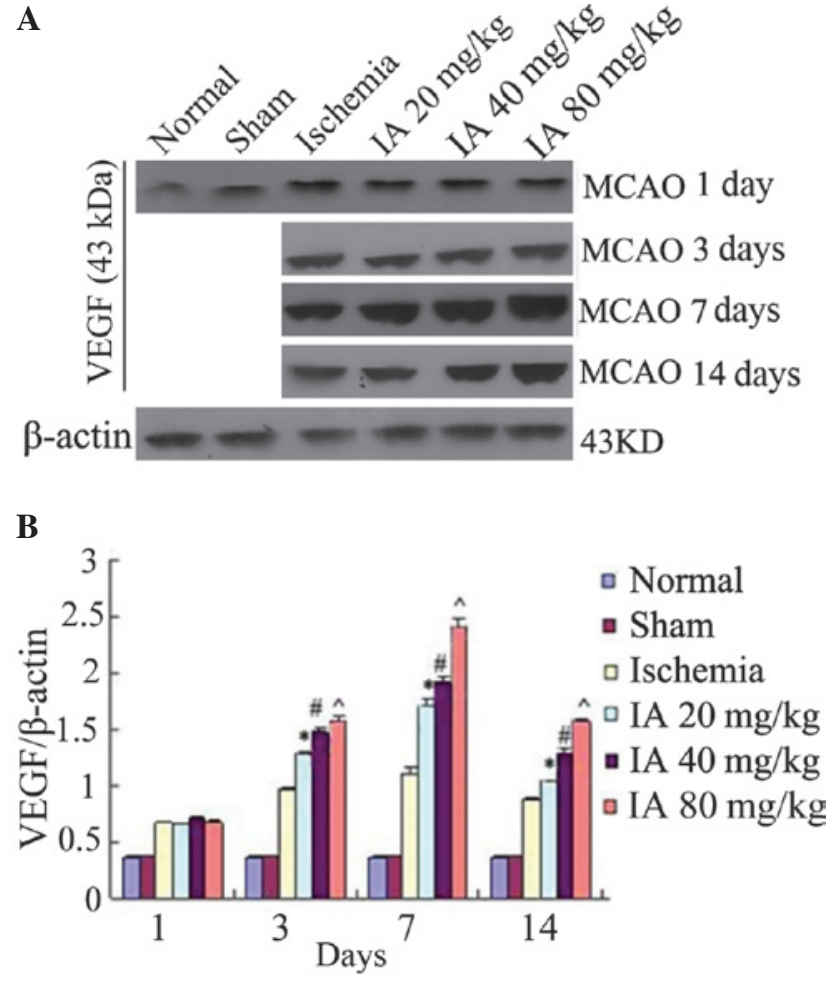

Figure 6. IA increases the expression of VEGF. Expression levels of VEGF was detected using (A) Western blot analysis and (B) results were quantified. $\beta$-actin was used as a loading control. Values are expressed as the mean \pm standard deviation $(\mathrm{n}=6)$. ${ }^{*} \mathrm{P}<0.05,{ }^{\#} \mathrm{P}<0.05$ and ${ }^{\wedge} \mathrm{P}<0.05$, vs. ischemia group. MCAO, middle cerebral artery occlusion; IA, Ilexonin A; VEGF, vascular endothelial growth factor.

circulation (3). In the present study, it was observed that Ilexonin A effectively reduced infarct volume and improved neurological deficits, in a dose-dependent manner, which demonstrated that Ilexonin A was a neuroprotectant. Previous studies have suggested that the neuroprotective effect of Ilexonin A may include enhancing the secretion of endogenous neural trophic factors, including FGF and GAP-43, promoting neurogenesis in the neocortical and subependymal zones (SVZs), mitigating cerebral edema, inhibiting free radical production and lipid peroxidation following cerebral ischemia/reperfusion $(4,5)$. However, the specific mechanism underlying the neuroprotective effects of Ilexonin A remain to be fully elucidated and requires further investigation.

The astrocyte response to ischemia in adults is complex and remains to be fully elucidated. A previous study found that reactive astrocytes with nuclear membranes increase cell size and number following MCAO (21). It is accepted that the moderate activation and proliferation of astrocytes can have a protective effect following ischemia/reperfusion; the protective mechanism of reactive astrocytes may be the result of multiple aspects. Previous studies have suggested that reactive astrocytes can acquire stem cell properties following injury and, thus, alter cell type to initiate repair following brain injury $(22,23)$. Reactive astrocytes are involved in neurovascular remodeling following ischemia/reperfusion $(24,25)$, and they also release $17 \beta$-estradiol; neurotrophic factors, including glia-derived neurotrophic factor; neurotrophin receptors, including tropomyosin receptor kinase $\mathrm{B}$; and
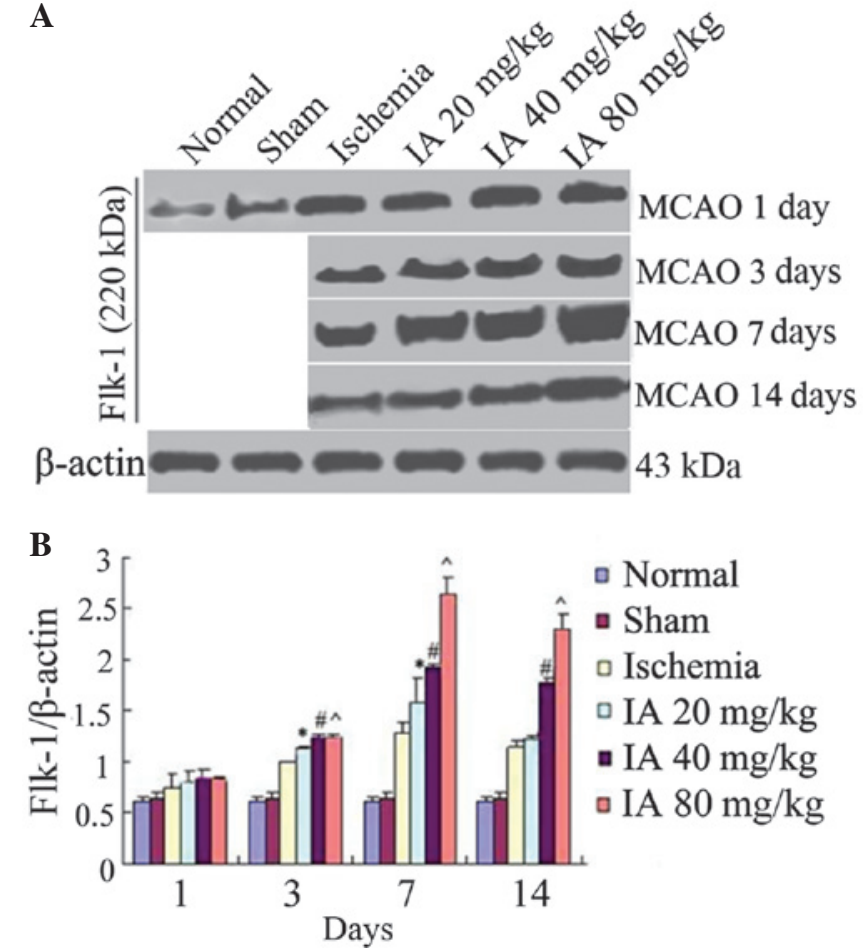

Figure 7. IA increases the expression of Flk-1. Expression levels of Flk-1 were detected using (A) Western blot analysis and (B) results were quantified. $\beta$-actin was used as a loading control. Values are expressed as the mean \pm standard deviation $(\mathrm{n}=6)$. ${ }^{*} \mathrm{P}<0.05,{ }^{\#} \mathrm{P}<0.05$ and ${ }^{\wedge} \mathrm{P}<0.05$, vs. ischemia group. MCAO, middle cerebral artery occlusion; IA, Ilexonin A; Flk-1, fetal liver kinase-1.

antioxidants to promote repair (26-29). If the activation of astrocytes is attenuated, the infarct volume expands 2-3-fold (30), and neuronal death is further enhanced following brain ischemia (25). The axonal growth cones grow only when reactive astrocytes survive, whereas this does not happened if astrocytes are injured in the same area following ischemia injury $(31,32)$. These findings indicate that reactive astrocytes are essential for neuronal survival and regeneration. However, the glial scar, which is formed by the excessive proliferation of reactive astrocytes in the later stage following ischemia/reperfusion, rebuilds the integrity of the CNS, but inhibits axonal regeneration (33). In the present study, Ilexonin A either enhanced the activation of astrocytes in the early stage (1, 3 or 7 days post-MCAO) or inhibited the excessive proliferation of astrocytes in the later stage (14 days post-MCAO) in the peri-infarct region following ischemia/reperfusion. This dual effect indicated that Ilexonin A promotes favorable conditions for neuronal restoration.

Microglia are the resident immune cells in the CNS. When there is a stimulatory signal in the brain, for example cerebral ischemia, the microglia become activated and can undergo phenotypic and morphological changes (10). Based on the expression of immunocytochemical markers, the microglia become amoeba-like cells following ischemia/reperfusion, which are morphologically indistinguishable from blood-derived macrophages (34). Therefore, Iba-1 positive cells are likely derived from resident microglial and blood-derived macrophages. Novel data, based on the use 
of GFP radiation bone marrow-chimeric mice, suggests that blood-derived macrophages are a low percentage of the population of cells in the peri-infarct region at day 2 post-ischemia, peak at day 7 and decrease thereafter (35). By contrast, resident microglial cells are rapidly activated at day 1 post-ischemia. Notably, even at days 4 an 7 , the majority of macrophage-like cells remain resident microglia-derived cells $(36,37)$. In addition, previous reports have suggested that microglia expressing different phenotypes and morphologies have different functions when the brain receives different signals from different diseases; and the function (neuroprotective or neurotoxic) may be determined by the steady-state conditions among various microglial factors released into the microenvironment (38-42). A previous study also suggested that resident microglial and newly recruited macrophages assume a 'healthy' M2 phenotype in the 7 days following MCAO, which protects neurons against ischemic injury. This is followed by a transition to an 'unhealthy' M1 phenotype in the peri-infarct region, which exacerbates neuronal death during ischemic stroke (43). The dual and opposing roles of microglial/macrophages suggest that ischemic stroke therapy requires a focus on adjusting the balance between beneficial and detrimental microglia and macrophage responses, rather than simply suppressing microglia/macrophages.

In conclusion, the present study, found that the number of amoeba-like cells significantly decreased, whereas the number of rod-like cells increased following treatment with Ilexonin A. This indicated that the rod-like microglia, which appeared within the first 3 days of MCAO and were maintained by the administration of Ilexonin A may have a protective effect against cerebral ischemia/reperfusion injury. The neuroprotective effects of Ilexonin A may be achieved through adjusting the balance of microglia/macrophage responses. In previous studies, the protective effect of microglia/macrophages were reported to occur through reduction in the secretions of neuronal plasticity proteins and neurotrophic factors, including IGF-1 and GDNF (42-45). However, the evidence for this is limited, and further investigation is required.

In the present study, the expression of Nestin was induced on day 1 following MCAO, increased progressively until day 7, when the highest expression was observed, and was sustained for $>2$ weeks. These results are consistent with those of previous studies $(15,46)$. The astrocyte-like Nestin-positive cells observed around the ischemic area following 3 days of MCAO indicated a trend for neural stem cells to predominantly differentiate into astrocytes. By contrast, a previous study suggested that either endogenous or transplanted neural stem cells at the site of brain injury predominantly differentiated into gliocytes, with the exception of SVZs and subgranular zones (47). In addition, reactive astrocytes express the Nestin protein and assist in neural stem cell proliferation and differentiation $(48,49)$. Although neural stem cells that proliferate into the peri-infarct region predominantly differentiate into gliocytes, a number of newly matured neurons are observed in the striatum and cortex at later time points post-ischemia/reperfusion $(50,51)$. It has been suggested that newly matured neurons in the striatum and cortex may be associated with neuronal migration from the SVZ (52). Our previous study suggested that the number of newly matured neurons were increased in the cortex, and that neurogenesis and neuronal migration from the SVZ were increased following treatment with Ilexonin A (6).

VEGF, one of the most important angiogenic factors, is a double-edged sword in ischemic injury. VEGF is known as a potent mitogen for angiogenesis in the ischemic boundary, and is a neuroprotective factor involved in reducing infarct volume, improving behavioral recovery, and enhancing cortical, striatal and SVZ neurogenesis and neuromigration following cerebral ischemia in rats (53-55). In addition, VEGF acts as an instructive signal and induces responsive astroglial cells toward neuronal differentiation (56). However, VEGF is a potent vascular permeability factor, which may mediate a harmful response through blood-brain barrier leakage, brain edema, hemorrhage, aggravation of inflammatory responses and aberrant systemic hemodynamics (57-59). Therefore, a major challenge is to accelerate cerebral angiogenesis without exacerbating edema in the brain of stroke patients.

Previous studies have suggested that the results of VEGF administration are variable and are associated with the following aspects of treatment: i) Dose-effectiveness, in which there are dose-dependent effects when different doses are administrated by different methods, and excess VEGF administration may have a detrimental effect in functional recovery (60-62); ii) Route dependence, in which local VEGF administration consistently enhances neurologic recovery, whereas acute intravenous delivery exacerbates brain infarcts due to enhanced brain edema; suggesting exogenous VEGF can be directly neurotoxic (63); iii) Time-dependence, in which VEGF has a therapeutic time window within the first $3 \mathrm{~h}$ or following $24 \mathrm{~h}$ of transient MCAO (62-64); iv) Special receptors, in which VEGF is known to be rapidly induced following focal cerebral ischemia and to bind to two tyrosine kinase receptors, fms-like tyrosine kinase-1 (Flt-1) and fetal liver kinase-1 (Flk-1), on the surface of endothelial cells. These receptors are induced following ischemia and show a similar temporal pattern of expression, however, the post-ischemic increase of Flk-1 is higher than that of Flt-1 (65). A previous study suggested that activation of Flt-1, but not Flk-1, is sufficient to induce hyper-permeability by hypoxia and VEGF (66). The results of the present study suggested that VEGF and Flk-1, which were progressively increased following transient focal cerebral ischemia and administration of Ilexonin A, predominantly exerted neuroprotective effects, which may be dose-dependent.

According to the observation of the dose-dependent effects of Ilexonin A, the present study found that the dose of $40 \mathrm{mg} / \mathrm{kg}$ produced the optimal protective effects on the brains of rats following transient focal cerebral ischemia. This result is in accordance with previously published data from Zheng and Shi (6).

In conclusion, neurologic recovery is a complex process, which requires the integration of numerous intrinsic and extrinsic cues to establish intact circuits. No single factor alone is sufficient to support long-term neurologic recovery. The results of the present study suggested that Ilexonin A provided a favorable microenvironment for neurological recovery through regulating the activation of gliocytes, and promoting revascularization and neurogenesis. 


\section{Acknowledgements}

This study was supported by the Program for New Century Excellent Talents in Fujian Province University, China (grant no. NCETFJ-0704) and the Professorial Academic Development Foundation of Fujian Medical University (grant no. JS09014). The authors would like to thank Clarity Manuscript Consultants LLC for their assistance with manuscript editing.

\section{References}

1. Fu XC, Xu Z and Chen JJ: Effects of MDQR4 on cardiac function and hemodynamics in anesthetized dogs. Zhong Xi Yi Jie He Xin Nao Xue Guan Bing 9: 1471-1473, 2011 (In Chinese)

2. Li XR: Overview of research progresses on pharmacological effects Ilex phescens. Glob Trad Chin Med 9: 238-240, 2011.

3. Huang XW, You ZD, Chen J and Xian SX: Effect of Radix Ilecis Pubescentis on heart function and the miR133a expression in chronic heart failure rats. Zhong Yao Xin Yao Yu Lin Chuang Yao Li 25: 48-92, 2014 (In Chinese).

4. Hua HY and Li YY: The pharmacological effects of Ilexonin A. Chin J Med 8: 137-138, 2006.

5. Sheng HL, Dong XL and Jiang YF: Protective effects of Radix Ilicis Pubescentis extract on focal cerebral ischemia reperfusion injury in rats. Zhongguo Xin Yao Za Zh 18: 1020-1022, 2009 (In Chinese).

6. Zheng GY and Shi WQ: Influence of Ilexonin A on the expression of bFGF, GAP-43 and neurogenesis after cerebral ischemia-reperfusion in rats. Yao Xue Xue Bao 46: 1065-1071, 2011 (In Chinese).

7. Kim DH, Kim S, Jung WY, Park SJ, Park DH, Kim JM, Cheong JH and Ryu JH: The neuroprotective effects of the seeds of Cassia obtusifolia on transient cerebral global ischemia in mice. Food Chem Toxicol 47: 1473-1479, 2009

8. Lee CH, Yoo KY, Hwang IK, Choi JH, Park OK, Li H, Kang IJ, Kwon YG, Kim YM and Won MH: Hypothyroid state does not protect but delays neuronal death in the hippocampal CA1 region following transient cerebral ischemia: Focus on oxidative stress and gliosis. J Neurosci Res 88: 2661-2668, 2010.

9. Villapol S, Gelot A, Renolleau S and Charriaut-Marlangue C: Astrocyte responses after neonatal ischemia: The yin and the yang. Neuroscientist 14: 339-344, 2008.

10. Badoer E: Microglia: Activation in acute and chronic inflammatory states and in response to cardiovascular dysfunction. Int J Biochem Cell Biol 42: 1580-1585, 2010.

11. Wang N, Zhang Y, Wu L, Wang Y, Cao Y, He L, Li X and Zhao J: Puerarin protected the brain from cerebral ischemia injury via astrocyte apoptosis inhibition. Neuropharmacology 79: 282-289, 2014

12. Shen Y, Sun A, Wang Y, Cha D, Wang H, Wang F, Feng L, Fang $S$ and Shen Y: Upregulation of mesencephalic astrocyte-derived neurotrophic factor in glial cells is associated with ischemia-induced glial activation. J Neuroinflammation 9 : 254, 2012.

13. Yenari MA, Xu L, Tang XN, Qiao Y and Giffard RG: Microglia potentiate damage to blood-brain barrier constituents: improvement by minocycline in vivo and in vitro. Stroke 37: 1087-1093, 2006.

14. Yang Z, Zhong L, Zhong S, Xian R and Yuan B: Hypoxia induces microglia autophagy and neural inflammation injury in focal cerebral ischemia model. Exp Mol Pathol 98: 219-224, 2015.

15. Shin HY, Kim JH, Phi JH, Park CK, Kim JE, Kim J-H, Paek SH, Wang KC and Kim DG: Endogenous neurogenesis and neovascularization in the neocortex of the rat after focal cerebral ischemia. J Neurosci Res 86: 356-367, 2008.

16. Navaratna D, Guo S, Arai K and Lo EH: Mechanisms and targets for angiogenic therapy after stroke. Cell Adh Migr 3: 216-223, 2009.

17. Mac Gabhann F, Qutub AA, Annex BH and Popel AS: Systems biology of pro-angiogenic therapies targeting the VEGF system. Wiley Interdiscip Rev Syst Biol Med 2: 694-707, 2010.

18. Li X, Zhang J and Liang SD: Function and mechanism of VEGF in the nervous system. Shenjing Jiepouxue Zazhi 26: 561-563, 2010 (In Chinese).
19. Longa EZ, Weinstein PR, Carlson S and Cummins R: Reversible middle cerebral artery occlusion without cranicetomy in rats. Stroke 20: 84-91, 1989

20. Abedin V, Toctam S and Mahdi Z: Evaluation the protective effect of aminoguanidine on cortex and striatum damage in acute phase of focal cerebral ischemia in rat. Physiol Pharmacol 11: 38-43, 2007.

21. Chu X, Fu X, Zou L, Qi C, Li Z, Rao Y and Ma K: Oncosis, the possible cell death pathway in astrocytes after focal cerebral ischemia. Brain Res 1149: 157-164, 2007.

22. Buffo A, Rite I, Tripathi P, Lepier A, Colak D, Horn AP, Mori T and Götz M: Origin and progeny of reactive gliosis: A source of multipotent cells in the injured brain. Proc Natl Acad Sci USA 105: 3581-3586, 2008

23. Sirko S, Behrendt G, Johansson PA, Tripathi P, Costa M, Bek S, Heinrich C, Tiedt S, Colak D, Dichgans M, et al: Reactive glia in the injured brain acquire stem cell properties in response to sonic hedgehog. Cell Stem Cell 12: 426-439, 2013.

24. Hayakawa K, Nakano T, Irie K, Higuchi S, Fujioka M, Orito K, Iwasaki K, Jin G, Lo EH, Mishima K and Fujiwara M: Inhibition of reactive astrocytes with fluorocitrate retards neurovascular remodeling and recovery after focal cerebral ischemia in mice. J Cereb Blood Flow Metab 30: 871-882, 2010.

25. Jing L, Mai L, Zhang JZ, Wang JG, Chang Y, Dong JD, Guo FY and Li PA: Diabetes inhibits cerebral ischemia-induced astrocyte activation - an observation in the cingulate cortex. Int J Biol Sci 9: 980-988, 2013.

26. Lin CH, Cheng FC, Lu YZ, Chu LF, Wang CH and Hsueh CM: Protection of ischemic brain cells is dependent on astrocyte-derived growth factors and their receptors. Exp Neurol 201: 225-233, 2006.

27. Tonchev AB, Boneva NB, Kaplamadzhiev DB, Kikuchi M, Mori Y, Sahara S and Yamashima T: Expression of neurotrophin receptors by proliferating glia in postischemic hippocampal CA1 sector of adult monkeys. J Neuroimmunol 205: 20-24, 2008.

28. Ding J, Yu JZ, Li QY, Wang X, Lu CZ and Xiao BG: Rho kinase inhibitor Fasudil induces neuroprotection and neurogenesis partially through astrocyte-derived G-CSF. Brain Behav Immun 23: 1083-1088, 2009.

29. Zhang QG, Wang R, Tang H, Dong Y, Chan A, Sareddy GR, Vadlamudi RK and Brann DW: Brain-derived estrogen exerts anti-inflammatory and neuroprotective actions in the rat hippocampus. Mol Cell Endocrinol 389: 84-91, 2014.

30. Li L, Lundkvist A, Andersson D, Wilhelmsson U, Nagai N, Pardo AC, Nodin C, Ståhlberg A, Aprico K, Larsson K, et al: Protective role of reactive astrocytes in brain ischemia. J Cereb Blood Flow Metab 28: 468-481, 2008.

31. Jeong HK, Jou I and Joe EH: Absence of delayed neuronal death in ATP-injected brain: Possible roles of astrogliosis. Exp Neurobiol 22: 308-314, 2013.

32. Takano T, Oberheim N, Cotrina ML and Nedergaard M: Astrocytes and Ischemic Injury. Stroke 40: 8-12, 2009.

33. Davies SJ, Goucher DR, Doller C and Silver J: Robust regeneration of adult sensory axons in degenerating white matter of the adult rat spinal cord. J Neurosci 19: 5810-5822, 1999.

34. Garcia JA, Cardona SM and Cardona AE: Analyses of microglia effector function using CX3CR1-GFP knock-in mice. Methods Mol Biol 1041: 307-317, 2013.

35. Schilling M, Besselmann M, Leonhard C, Mueller M, Ringelstein EB and Kiefer R: Microglial activation precedes and predominates over macrophage infiltration in transient focal cerebral ischemia: A study in green fluorescent protein transgenic bone marrow chimeric mice. Exp Neurol 183: 25-33, 2003.

36. Lalancette-Hébert M, Gowing G, Simard A, Weng YC and Kriz J: Selective ablation of proliferating microglial cells exacerbates ischemic injury in the brain. J Neurosci 27: 2596-2605, 2007.

37. Breckwoldt MO, Chen JW, Stangenberg L, Aikawa E, Rodriguez E, Qiu S, Moskowitz MA and Weissleder R: Tracking the inflammatory response in stroke in vivo by sensing the enzyme myeloperoxidase. Proc Natl Acad Sci USA 105: 18584-18589, 2008.

38. Nakajima K and KoItsaka S: Microglia: Neuroprotective and neurotrophic cells in the central nervous system. Curr Drug Targets Cardiovasc Haematol Disord 4: 65-84, 2004.

39. Kim SU and de Vellis J: Microglia in health and disease. J Neurosci Res 81: 302-313, 2005.

40. Li L, Lua J, Taya SS, Moochhalab SM and Hea BP: The function of microglia, either neuroprotection or neurotoxicity, is determined by the equilibrium among factors released from activated microglia in vitro. Brain Res 1159: 8-17, 2007. 
41. Ekdahl CT, Kokaia Z and Lindvall O: Brain inflammation and adult neurogenesis: The dual role of microglia. Neuroscience 158: 1021-1029, 2009.

42. Wang JP, Yang ZT, Liu C, Zhao YZ and Chen YB. Activated microglia provide a neuroprotective role by balancing glial cell-line derived neurotrophic factor and tumor necrosis factor- $\alpha$ secretion after subacute cerebral ischemia. Int J Mol Med 31: 172-178, 2013.

43. Hu X, Li P, Guo Y, Wang H, Leak RK, Chen S, Gao Y and Chen J: Microglia/macrophage polarization dynamics reveal novel mechanism of injury expansion after focal cerebral ischemia. Stroke 43: 3063-3070, 2012.

44. Arroba AI, Alvarez-Lindo N, van Rooijen N and de la Rosa EJ: Microglia-mediated IGF-I neuroprotection in the rd10 mouse model of retinitis pigmentosa. Invest Ophthalmol Vis Sci 52: 9124-9130, 2011.

45. Montero M, González B and Zimmer J: Immunotoxic depletion of microglia in mouse hippocampal slice cultures enhances ischemia-like neurodegeneration. Brain Res 1291: 140-152, 2009.

46. Liu W, Song DL, Zhang GH, Chen JW, Xie RH, Lin HJ, et al: Nestin expression in different area of brain after cerebral ischemia/reperfusion in rats. Zu Zhong Yu Shen Jing Ji Bing 16 34-36, 2009.

47. Song H, Stevens CF and Gage FH: Astroglia induce neurogenesis from adult neural stem cells. Nature 417: 39-44, 2002.

48. Shin YJ, Kim HL, Park JM, Cho JM, Kim SY and Lee MY: Characterization of nestin expression and vessel association in the ischemic core following focal cerebral ischemia in rats. Cell Tissue Res 351: 383-395, 2013.

49. Kronenberg G, Gertz K, Cheung G, Buffo A, Kettenmann H, Gotz $\mathrm{M}$ and Endres M: Modulation of fate determinants Olig2 and Pax6 in resident glia evokes spiking neuroblasts in a model of mild brain ischemia. Stroke 41: 2944-2949, 2010.

50. Kim HJ, Leeds P and Chuang DM: The HDAC inhibitor, sodium butyrate, stimulates neurogenesis in the ischemic brain. J Neurochem 110: 1226-1240, 2009.

51. Yao RQ, Zhang L, Wang W and Li L: Cornel iridoid glycoside promote neurogenesis and angiogenesis and improves neurological function after focal cerebral ischemia in rats. Brain Res Bull 79: 69-76, 2009

52. Zhong J, Tang MK, Zhang Y, Xu QP and Zhang JT: Effect of salvianolic acid $\mathrm{B}$ on neural cells damage and neurogenesis after brain ischemia-reperfusion in rats. Yao Xue Xue Bao 42: 716-721, 2007 (In Chinese).

53. Wang YQ, Cui HR, Yang SZ, Sun HP, Qiu MH, Feng XY and Sun FY: VEGF enhance cortical newborn neurons and their neurite development in adult rat brain after cerebral ischemia. Neurochem Int 55: 629-636, 2009.

54. Yang JP, Liu HJ and Liu XF: VEGF promotes angiogenesis and functional recovery in stroke rats. J Invest Surg 23: 149-155, 2010 .
55. Herz J, Reitmeir R, Hagen SI, Reinboth BS, Guo Z, Zechariah A ElAli A, Doeppner TR, Bacigaluppi M, Pluchino S, et al: Intracerebroventricularly delivered VEGF promotes contralesional corticorubral plasticity after focal cerebral ischemia via mechanisms involving anti-inflammatory actions. Neurobiol Dis 45: 1077-1085, 2012.

56. Li P, Huang JJ, Ni JJ and Sun FY: WITHDRAWN: VEGF evokes reactive astroglia to convert into neuronal cells by affecting the biological function of $\mathrm{MeCP} 2$ in adult rat brain after cerebral ischemia. Neurochem Int, 2012 (Epub ahead of print).

57. Wang Y, Kilic E, Kilic U, Weber B, Bassetti CL, Marti HH and Hermann DM: VEGF overexpression induces post-ischaemic neuroprotection, but facilitates haemodynamic steal phenomena. Brain 128: 52-63, 2005.

58. Chibay Y, Sasayama T, Miyake TS, Koyama J, Kondoh T, Hosoda K and Kohmura E: Anti-VEGF receptor antagonist (VGA1155) reduces infarction in rat permanent focal brain ischemia. Kobe J Med Sci 54: E136-E146, 2008.

59. Li Z, Wang R, Li S, Wei J, Zhang Z, Li G, Dou W, Wei Y and Feng M: Intraventricular pre-treatment with rAAV-VEGF induces intracranial hypertension and aggravates ischemic injury at the early stage of transient focal cerebral ischemia in rats. Neurol Res 30: 868-875, 2008.

60. Manoonkitiwongsa PS, Schultz RL, McCreery DB, Whitter EF and Lyden PD: Neuroprotection of ischemic brain by vascular endothelial growth factor is critically dependent on proper dosage and may be compromised by angiogenesis. J Cereb Blood Flow Metab 24: 693-702, 2004.

61. Katsumata A, Sugiu K, Tokunaga K, Kusaka N, Watanabe K, Nishida A, Namba K, Hamada H, Nakashima $H$ and Date I: Optimal dose of plasmid vascular endothelial growth factor for enhancement of angiogenesis in the rat brain ischemia model. Neurol Med Chir (Tok yo) 50: 449-455, 2010.

62. Yang JP, Guo L, Liu RC and Liu HJ: Neuroprotective effects of VEGF administration after focal cerebral ischemia/reperfusion: Dose response and time window. Neurochem Int 60: 592-596, 2012.

63. Hermann DM and Zechariah A: Implications of vascular endothelial growth factor for postischemic neurovascular remodeling. J Cereb Blood Flow Metab 29: 1620-1643, 2009.

64. Sun Y, Jin K, Xie L, Childs J, Mao XO, Logvinova A and Greenberg DA: VEGF-induced neuroprotection, neurogenesis and angiogenesis after focal cerebral ischemia. J Clin Invest 111: $1843-1851,2003$

65. Choi JS, Kim HY, Cha JH, Choi JY, Chun MH and Lee MY: Upregulation of vascular endothelial growth factor receptors Flt-1 and Flk-1 in rat hippocampus after transient forebrain ischemia. J Neurotrauma 24: 521-531, 2007.

66. Vogel C, Bauer A, Wiesnet M, Preissner K, Schaper W, Marti HH and Fischer S: Flt-1, but not Flk-1 mediates hyperpermeability through activation of the PI3-K/Akt pathway. J Cell Physiol 212: 236-243, 2007. 